\title{
Simulation-based Analysis and Internet-based Realisation of Collaborative Product Development Processes
}

\author{
Prof. Dr.-Ing. F.-L. Krause \\ Dipl.-Ing. M. Doblies \\ Dipl.-Ing. C. Raupach \\ Fraunhofer-Institute for Production Systems and Design \\ Technology - IPK Berlin \\ Pascalstr. 8-9, D-10587 Berlin, Germany \\ Fax-no.: $\quad++49303930246$ \\ e-mail:_frank-l.krause@ipk.fhg.de
}

\begin{abstract}
The combination of computer-aided tools for the support of planning and execution of product development processes opens new possibilities especially for the collaboration in virtual enterprises and globally distributed supply chains. The use of simulation tools for process analysis not only enables the enterprises to design optimised product development processes across company borders but can also form the basis for the execution of these processes. The support granted by product library and workflow-management functionalities ensures that planned processes are carried out in an effective way without struggling with the usual information management problems.

The described approach of a continuous support of the design and execution of product development processes helps companies to face the challenges of a globalised world by using the opportunities that such a globalised world offers.
\end{abstract}

Keywords

product development processes, simulation, distributed design, product data management, 


\section{INTRODUCTION}

The ongoing globalisation in the world of manufacturing industries has two major impacts on the product development processes forming both a challenge and a solution for the new situation. The globalisation of the market place creates the need of very effective product development processes in order to react quickly on market changes. On the other hand the globalisation of the manufacturing industries enables enterprises to use globally distributed supply chains (Spur-97).

Re-engineering the product development processes taking into account the changed situation on the world-market has to aim at improving the agility of the company. This is especially true if the second effect of global supply chains and co-operations can be included in the re-organisation of the processes. However, the complexity of product development processes, especially those which are carried out in distributed locations leading to so-called Virtual Enterprises is immense This makes it imperative to use computer-aided tools for the planning, organisation and realisation of the distributed product development processes. At IPK Berlin several tools of this kind were developed in order to support distributed product development processes from planning to execution.

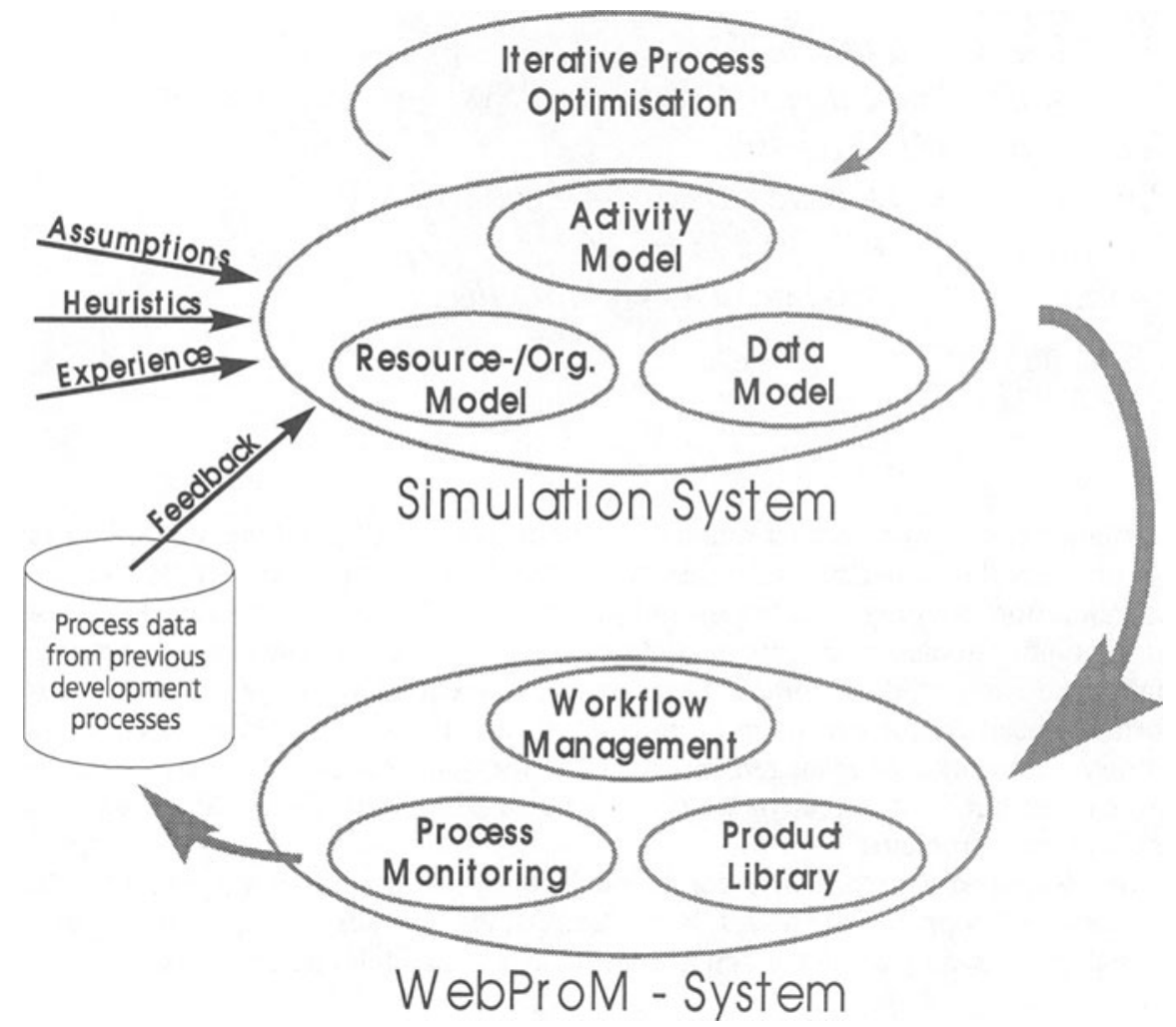

Figure 1. Linkage between simulation system and WebProM 
The simulation system is used to identify weak-points in the existing or planned development process. For this simulation certain assumptions for characteristic values such as typical processing time for tasks or probabilities for iterative loops have to be taken. Although this seems to be difficult the involved developers usually can give relative good assumptions based on their experience from earlier development processes. The results from the process optimisation can be used to define the product data structure and the workflow-support for the execution of the development process. The system supporting the execution of the development process monitors the activities and gives feedback to the simulation systems in terms of values supporting the build-up of future development process models. This feedback adds to the assumptions and heuristics and by this improves the quality of the simulation model (Figure 1).

\section{SIMULATION-BASED ANALYSIS OF PRODUCT DEVELOPMENT PROCESSES}

Very often product development processes are seen as a unique unpredictable sequence of highly creative tasks (Adler 1995). Product development processes would then not be plannable because the process course is evolving only during the process based on the results of the process. A sensible planning or even simulation would then be impossible. But these assumptions are - if at all - only true for complete new developments of highly innovative products. In reality, however most development processes are based on already existing predecessors of the product and can be characterised rather as adaptive of variant design. Therefore usually the companies have a rather good descriptions or plans of the development process.

When planning and organising processes as complex as those in product development it is virtually impossible to completely understand the entire system. One of the most effective means for analysing highly complex and dynamic systems has proven to be simulation technique. By using a process simulation model with the appropriate parameters it becomes possible to quantitatively estimate the effects of changes in the process organisation in much more detail than by pure heuristic analysis, which is currently the most common approach in development process planning. Influential parameters can be identified and the overall process can be optimised according to the appropriate optimisation criteria.

The simulation system developed at IPK Berlin in the course of the Fraunhofer research initiative DZ-SIMPROLOG consists of three partial models (Krause-98). The activity model specifies the activities to be conducted during the process, their logical order, time aspects and resource requirements. The resource and organisational model reflects the organisational structure of the enterprise and the human and technical resources allocated to the organisational units. The data model monitors the development of product data as it evolves during the simulated development process.

Results from the simulation runs can be used to identify weak points and optimisation potentials of the development process. Appropriate means to improve the process performance can be applied and their effectiveness can be evaluated using the simulation system (Figure 2). 
The activity model describes not only the conventional forward-oriented workflow but also the backward-oriented workflow caused by iterative loops, ramifications and backward leaps. Since the development process is not deterministic but has a high degree of uncertainty for the occurrence of the iterations, and processing time of the activities, statistical values are introduced which reflect the possible process variations. This is of particular importance for product development processes The activities themselves are described by characteristic parameters such as processing time, cost or resource requirements.

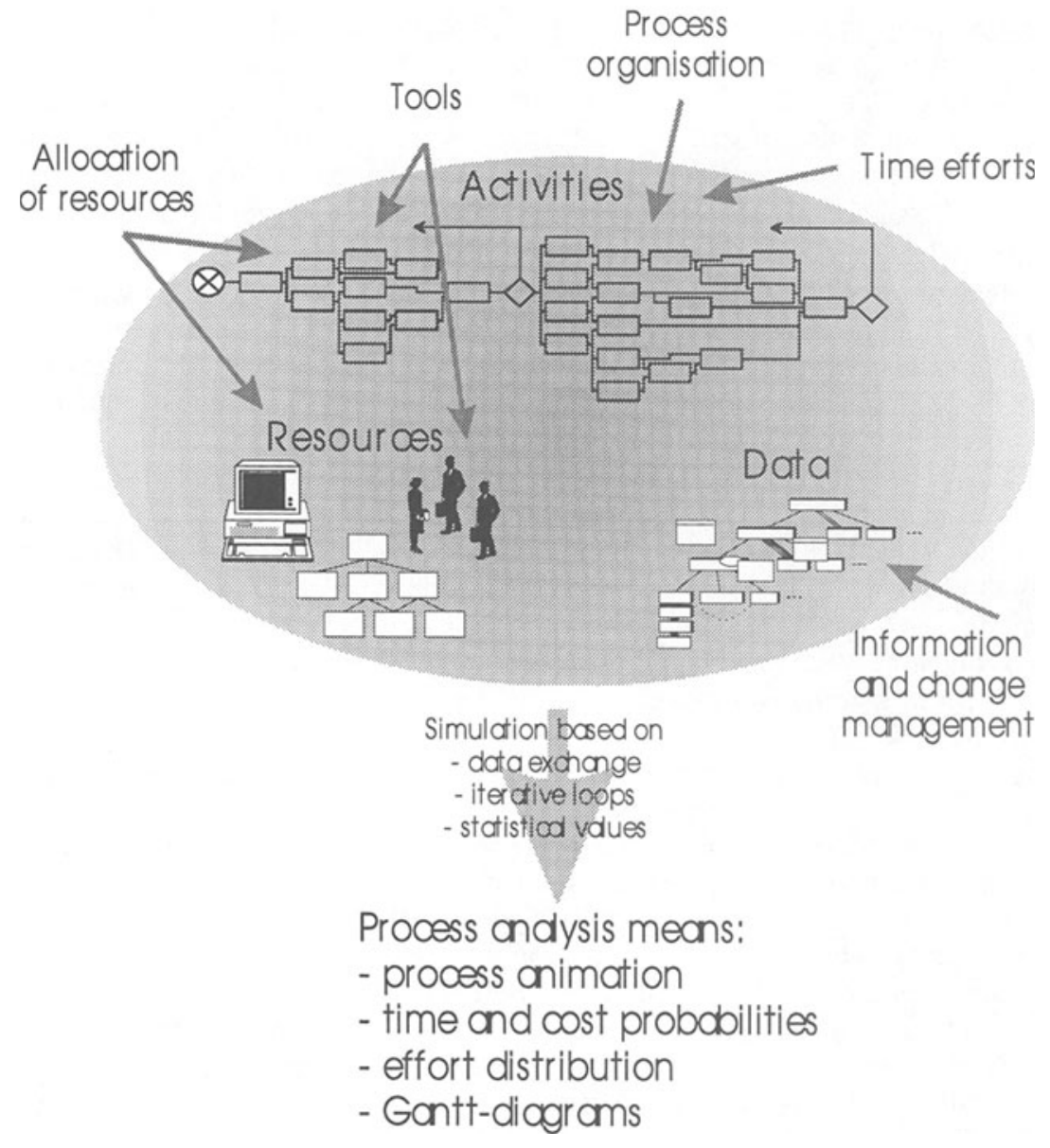

Figure 2. Modelling and simulation of the product development process.

The resource and organisation model defines the amount and capabilities of the resources which are available to the development processorganisation. Resources include both technical and human resources. The people involved in the product development process are described with their qualification. This qualification can be divided into process-specific qualification, describing what tasks can be 
performed by the people, and system-specific qualification, representing the capabilities to use certain IT-tools. During the simulation run the resource and organisation model guarantees that resources cannot be used by different activities in parallel.

The third model, the data model makes it possible to monitor in which sequence product data is created and when certain data is required. Additionally it becomes possible to identify whether and how often activities are using data which is outdated by later versions of data documents. This function offers the process planner the possibility to quantitatively evaluate the usefulness of adequate change and information management as given by a properly maintained PDM-system or a web-based product library system like WebProM described below.

The simulation of the product development process is carried out by a discrete simulation using time-based Petri-nets. A development order is created and propagated through the activity network of the activity model. Data documents are created as objects in the activities and can be traced in order to derive cost and time values for single tasks, part processes or the entire development process.

Depending on the level of detail of the process model the simulation system can analyse the effectiveness of existing and planned processes according to the applied development method such as Simultaneous Engineering or Lean Development. Resource allocation can be optimised considering the not precisely predictable course of events and the effects of parallel, competing processes. The document objects exchanged between the activities have a creation date and a version number, so that it becomes possible to monitor the consistency of the data and thereby the management of change. By defining different ways of communication and collaboration both between activities performed within one company and between several companies the aspects of distributed product development processes can be modelled and analysed. As an example modern communication means such as teleconferences, multimedia-mail or shared applications can be compared with more conservative means such as physical meetings, telephone and faxes.

The outcome of the simulation-based optimisation of the product development processes is a very mature process description which can be used as a basis for the following execution of the product development process. Another major result is the description of the product data structure which can be used for the organisation of the product model data to be used during the product development.

\section{INTERNET-BASED EXECUTION OF COLLABORATIVE PRODUCT DEVELOPMENT}

Due to the requirements of collaborative distributed product development processes IPK has developed a WWW-based Product and Project Library for the support of co-operation between different companies. By storing, retrieving and maintaining up-to-date data in a coherent structure the respective product data is accessible by the appropriate project partners and by the use of authorisation mechanisms to them only. The architecture of the product library is based on the management of files and folders. Using World Wide Web technology the support of various different file formats is limited only by the availability of the necessary 
browsers. This results in a high system flexibility. Being based on TCP/IP, the system can be used both in enterprise-wide TCP/IP-based Intranets as well as in cross-company Extranets or the Internet (Figure 2). Using highly sophisticated encryption methods the system can be used even via public Internet routes with a very high degree of security, enabling world-wide product development collaboration even with extremely sensitive data.

By exploiting the combined power of HTML, CGI, JavaScript and Java the implemented system provides the user with a GUI that is oriented towards common applications. This makes the system very easily usable, especially for users who work normally with other software and use this library only as a complementary tool.

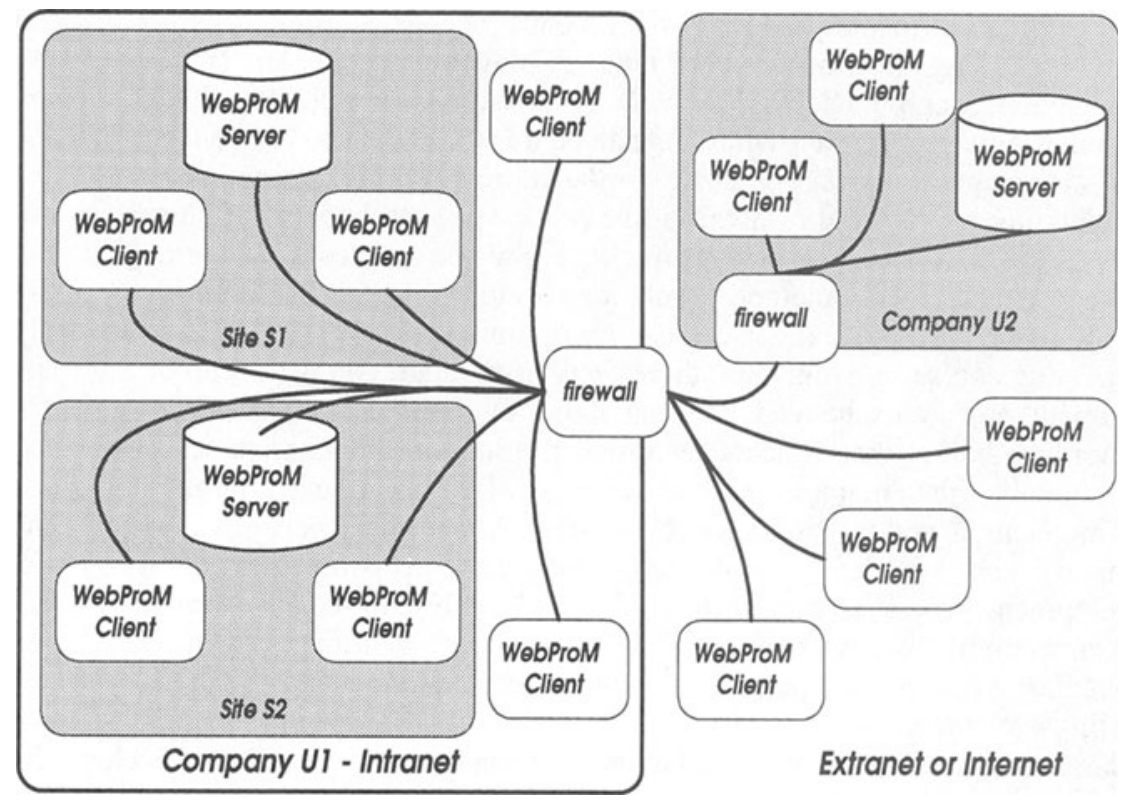

Figure 3. Data exchange in Intranet and Extranet.

By supplementing the capabilities of the Product Library with workflow management functionalities the execution of the development process can be effectively supported. A special hurdle is to implement one workflow system in several different companies. By providing the workflow support via Web technologies like HTML, JavaScript and Java this becomes possible. Browsers supporting these technologies can be found on most computers, resulting in a broad base of pre-installed workflow clients.

The workflow description of the simulation system forms the basis of the organised execution of the processes. The information from the data model of the simulation system is the main input for the build-up of the data and file structure of the product library. Access rights as well as information procedures are derived directly from the simulation system. Different kinds of releases of the elaborated 
product descriptions are spread out according to the description in the simulation system using the functionalities of both the product library and the workflow mechanisms. The timely constraints derived from the process simulation can be used also for project monitoring and management.

Furthermore this data exchange environment makes monitoring the real flow of information easy to achieve, thereby enabling a validation method for the simulation model. Measurements, including iterations and on-the-fly changes to the predefined workflow, are stored in an extended to the simulation model. After having collected a fair amount of measurements, these can be used to fine-tune the estimates used to create the simulation model. However, it has to be noted, that an automated evaluation of workflows, which consist mainly of exceptions, is currently not possible. The automated isolation of workflow components and the automated deduction of corresponding simulation parameters is a field with vast potential for research.

The measurements pose severe security problems as soon as information flow inside other companies is measured (Figure 4). Data security of the information being exchanged is achieved by the security mechanisms built into the WebProM system, like user authentification, access authorisation on a folder and user group level and strong encryption between different sites (Krause 1996). A similar security concept must be available to guarantee that external partners cannot look into the company's processes without special permission. Default behaviour is to clip measurements at company borders, thereby aggregating all workflows inside the company and presenting the aggregation as a black box to outsiders.

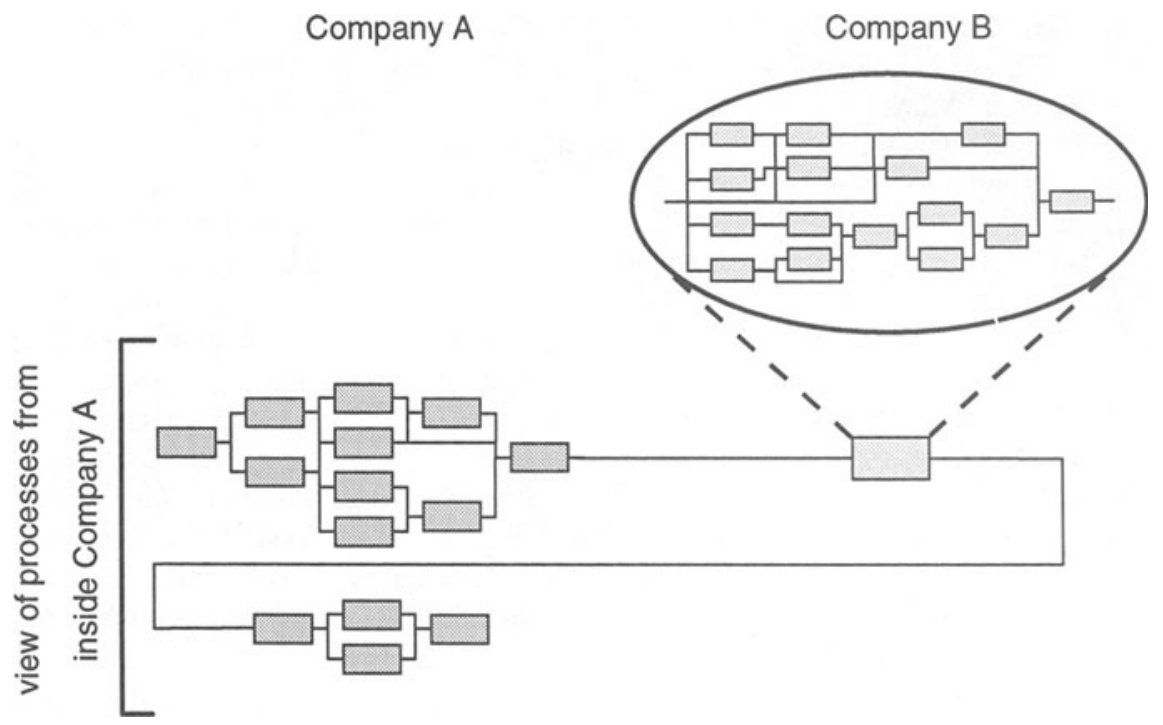

Figure 4. Security in company-crossing workflows using different levels of detail. 


\section{SUMMARY}

Product development processes can gain a great improvement if not only the single tasks are supported by information technology but also the process view on development is taken into consideration. The overall process structure can be modelled in the simulation system. Using various process analysis means such as Gantt-diagrams or animation process weakpoints can be identified and the process is optimised by the process planner using both efficiency and effectiveness improvement of the process. Possible means are modifications of the workflow, structural activity reorganisation, allocation of additional resources or the use of advanced information technology.

After the optimisation the resulting process description can be used as a basis for the definition of the workflow in the project and product library WebProM. Another outcome of the process modelling and simulation is a description of the data structure of the used product model. Again this structure is used in the WebProM system for the creation of the web-based product library. During the execution characteristic process data such as processing times are monitored in order to improve the modelling accuracy of future development projects. The webbased technology of WebProM enables the participating development partners to work co-operatively without sharing sensitive data. Not even the detailed process structure are revealed to the development partners. Therefore effective cooperation can be guaranteed without touching security issues which is especially interesting in the sensitive area of product development.

\section{REFERENCES}

Adler, P.S., Mandelbaum, A., Nguyen, V. and Schwerer, E. (1995) From Project to Process Management: An Empirically-based Framework for Analyzing Product Development Time. In: Management Science, Vol. 41, No.3, März 1995, pp. 458 - 484

Krause, F.-L. and Doblies, M. (1996) Sicherheitsaspekte Internet-basierter Informationssysteme. In: ZWF 91 (1996) 7-8. Carl Hanser Verlag, München 1996, pp. 344-346.

Krause, F.-L. and Doblies, M. (1997) A Communication Environment For Global Automotive Engineering. In: Proceedings of International Symposium on Global Engineering Networking, Antwerpen 22-24.4.1997. HNI-Verlagsschriftenreihe Band 21, Paderborn, pp 211-226.

Krause, F.-L. and Raupach, C (1998) Simulation of Product Development Processes. Proceedings of the First International Workshop on Intelligent Manufacturing Systems, 15-17 April 1998, Lausanne, Switzerland.

Spur, G. and Krause, F.-L. (1997) Das virtuelle Produkt - Management der CADTechnik. Hanser, München, Wien.

\section{BIOGRAPHY}

Prof. Dr.-Ing. Frank-Lothar Krause, born 1942, studied Production Technology at the Technical University Berlin. In 1976, he became Senior Engineer for the CAD Group at the Institute for Machine Tools and Production Technology (IWF) 
of the TU Berlin and earned his doctorate under Prof. Spur. Since 1977, he has been Director of the Design Technology Department at the Fraunhofer Institute for Production Systems and Design Technology (IPK Berlin). He earned the qualification as a university lecturer in 1979 and has been University Professor for Industrial Information Technology at the IWF of the TU Berlin since 1990.

Dipl.-Ing. Matthias Doblies, born 1965, studied aerospace engineering at the Technical University of Berlin. In 1994 he joined the department of Industrial Information Technologies at the Institute of Machine Tools and Manufacturing Technology of the Technical University of Berlin. He has worked in several national and international research projects in the field of telecommunications and geometric modeling.

Dipl.-Ing. Christoph Raupach, born in 1968, studied automotive engineering at the Technical University of Berlin. Since 1994 he is working at the Institute for Machine Tools and Factory Management of the Technical University of Berlin. He is working in the group modelling systems of the Department Industrial Information Technology. 